\title{
Dynamic Modeling of Generic Body Temperatures with Mean-Reverting Process and a Martingale Estimation Function
}

\author{
Hongliang Wang, Shimin Zhang
}

\begin{abstract}
In this paper a stochastic differential equation (SDE) model of generic body temperature (such as axilla, mouth, anus, etc.) fluctuation is developed. We consider a mean-reverting SDE process and use zero-mean martingale estimation function to get the parameters. Subsequently we use data generated from another dynamic model of core body temperature a ground truth for comparison with test our SDE model.
\end{abstract}

Keywords - Stochastic differential model, mean-reverting process, Fourier series, zero-mean martingale estimation function.

\section{INTRODUCTION}

$\mathbf{H}$ UMAN body temperature is not a static process but fluctuates throughout the day with amplitudes varying over time, and it is subject to many affections both endogenous (e.g. fitness, aging, hormones, and circadian) and exogenous (e.g. ambient temperature, diet, and lifestyle) [2]. Meanwhile, different measuring sites have varying temperature characteristics [2]. The present manuscript investigates the general body temperature, $T(t)$, which could be axillary, oral, core temperature, etc. Despite of all these uncertainties, human body temperature follows circadian rhythms, which means it varies diurnally with a nadir in the early morning, a zenith in the late afternoon, and a mean amplitude of variability of $0.5^{\circ} \mathrm{C}\left(0.9^{\circ} \mathrm{F}\right)[4],[1]$.

There are researches about dynamic modeling of body temperature such as [10] and [11] referred to in Section IV. However, what we are trying to do in the manuscript is to introduce the stochastic process, i.e. $W(t)$, into the simulation of body temperature. The result shows the feasibility.

The approach of the manuscript is to leverage a modified mean-reverting process. By assuming the body temperature, $T(t)$, satisfies a mean-reverting SDE, where the reverted mean function is assumed to be expressible as a Fourier series, we obtain $T(t)$ after solving the SDE with Itô's Formula. Section III details a method for detail the way to parameter estimation of $\theta$ and volatility $\sigma$ which uses martingale estimation function from [7] . To provide a quantitative evaluation of our method, an extant autoregressive model (with $\mathrm{AR}(1)$ ) [10], [11] is employed as a ground truth for comparison with our SDE model. We compare the results of the two models which turn out to be match in the general trend and indicate the

Hongliang Wang is now with University of South Florida, Tampa, FL, 33620 USA (e-mail:wangh1@ mail.usf.edu)

Shimin Zhang is now with Department of Public Health University of South Florida, Tampa, FL, 33612 USA (e-mail:shimin1@ mail.usf.edu)

Manuscript received October 30, 2019; revised Match 31, 2020. basic feasibility of our model in terms of portraying body temperature.

\section{General ASSUMPtions}

In this paper, we consider the human body temperature variation on an hourly basis. Let's assume the body temperature $T(t)$ to be the solution of the following stochastic differential equation (SDE):

$$
d T(t)=\theta(M(t)-T(t)) d t+\sigma(t) d W(t)
$$

where $\theta$, a parameter, determines the speed of mean-reversion, $M(t)$ is the mean to which the process reverts, and $W(t)$ is a Wiener process.

Notably, $M(t)$ has to be constant if (1) only reverts to $M(t)$ (Prop 4.1 [3]). However, $M(t)$ is a deterministic function that expresses the trend and seasonality of body temperature. To obtain a stochastic process reverting to $M(t)$ we add the term $\frac{d M(t)}{d t}$ to the drift term in (1). Now we get a model for body temperature is given as

$$
d T(t)=\left[\frac{d M}{d t}+\theta(M(t)-T(t))\right] d t+\sigma(t) d W(t)
$$

with solution

$T(t)=(T(0)-M(0)) e^{-\theta t}+M(t)+\int_{0}^{t} e^{-\theta(t-u)} \sigma(u) d W(u)$

which can be solved by Itô's Formula through, say, function $F$ satisfying $F\left(t, T_{t}\right)=T(t) e^{\theta t}$.

For the mean temperature $M(t)$ we use a Fourier series to depict body temperature's periodic characteristic. Additionally, it is assumed that there is no trend in $M(t)$, since we know body temperatures fluctuate around a fixed average value.

\section{PARAmeters Estimation}

\section{A. Estimation of the mean temperature function}

Write $M(t)$ in the form of a Fourier series as follows

$$
M(t)=a_{0}+\sum_{i=1}^{n}\left[a_{i} \cos (\omega i t)+b_{i} \sin (\omega i t)\right]
$$

where $\omega=\frac{2 \pi}{24}$ with assumed period of 24 hours. To estimate the parameters of the above function we apply the least square method. 


\section{B. Estimation of the volatility $\sigma$}

In this section we adopt the method of [5]. This method needs two estimators for $\sigma$. The first estimator is the quadratic variation of $T(t)$ :

$$
{\sigma_{1}}^{2}=\frac{1}{N} \sum_{i=0}^{N}(T(i+1)-T(i))^{2} .
$$

where $\mathrm{N}$ denotes the number of data points collected each day. The second estimator is derived by discretizing (2) which can be seen as a regression of the present temperature against previous moment temperature. After discretizing (2) becomes

$T_{i}=M_{i}-M_{i-1}+\theta M_{i-1}+(1-\theta) T_{i-1}+\sigma \epsilon_{i-1} \quad i=1, \ldots, N$ where $\left\{\epsilon_{i}\right\}_{i=1}^{N}$ are independent standard normally distributed random variables. Let $\hat{T}_{i} \equiv T_{i}-\left(M_{i}-M_{i-1}\right)$. Hence

$$
\hat{T}_{i}=\theta M_{i-1}+(1-\theta) T_{i-1}+\sigma \epsilon_{i-1} .
$$

By following [6] we obtain an efficient estimator of $\sigma$ as

$$
\sigma_{2}^{2}=\frac{1}{N-2} \sum_{i=1}^{N}\left(\hat{T}_{i}-\theta M_{i-1}-(1-\theta) T_{i-1}\right)^{2} .
$$

To complete the estimator in (6), an estimator for $\theta$ must be determined. This is the objective of the next section.

1) Estimation of parameter $\theta$ : One of the significant application of Girsanov theorem is to obtain an estimation of a function that satisfies $d T_{t}=\mu\left(T_{t} ; \theta\right)+\sigma\left(T_{t} ; \theta\right)$ as follows [7]

$$
\frac{d P}{d P_{w}}=\exp \left(\int_{0}^{t} \frac{\mu\left(T_{s} ; \theta\right)}{\sigma^{2}\left(T_{s}\right)} d T_{s}-\frac{1}{2} \int_{0}^{t} \frac{\mu^{2}\left(T_{s} ; \theta\right)}{\sigma^{2}\left(T_{s}\right)} d s\right)
$$

where $P$ and $P_{w}$ are the probability distributions of $T_{t}$ and Wiener process respectively. Using Itô and Riemann summations to approximate the above integral and differentiating with respect to $\theta$, we can get

$$
\sum_{i=1}^{n} \frac{\dot{\mu}\left(T_{i-1} ; \theta\right)}{\sigma^{2}\left(T_{i-1}\right)}\left(T_{i}-T_{i-1}\right)-\Delta \sum_{i=1}^{n} \frac{\mu\left(T_{i-1} ; \theta\right) \dot{\mu}\left(T_{i-1} ; \theta\right)}{\sigma^{2}\left(T_{i-1}\right)},
$$

where the dot denotes differentiation with respect to $\theta$ and $\Delta$ is the time interval length. When $\sigma$ does depend on $\theta$ the above estimation function becomes

$S_{n}=\sum_{i=1}^{n} \frac{\dot{\mu}\left(T_{i-1} ; \theta\right)}{\sigma^{2}\left(T_{i-1} ; \theta\right)}\left(T_{i}-T_{i-1}\right)-\Delta \sum_{i=1}^{n} \frac{\mu\left(T_{i-1} ; \theta\right) \dot{\mu}\left(T_{i-1} ; \theta\right)}{\sigma^{2}\left(T_{i-1} ; \theta\right)}$

. However, this estimating function is not consistent when the time between observations is bounded away from zero [8], and can be strongly biased when the time between observations is not small enough [9]. Here we apply the method of [7] to adjust (7) by subtracting its compensator in order to get a zero-mean martingale regarding the filtration defined by $\mathcal{F}_{i}=$ $\sigma\left(T_{1}, \ldots, T_{i}\right)$. The compensator is calculated as

$$
\begin{aligned}
& \sum_{i=1}^{n} E_{\theta}\left\{S_{i}-S_{i-1} \mid \mathcal{F}_{i-1}\right\} \\
&=\sum_{i=1}^{n} \frac{\dot{\mu}\left(T_{i-1} ; \theta\right)}{\sigma^{2}\left(T_{i-1} ; \theta\right)}\left(E_{\theta}\left(T_{i} \mid T_{i-1}\right)-T_{i-1}\right) \\
&-\Delta \sum_{i=1}^{n} \frac{\mu\left(T_{i-1} ; \theta\right) \dot{\mu}\left(T_{i-1} ; \theta\right)}{\sigma^{2}\left(T_{i-1} ; \theta\right)}
\end{aligned}
$$

. By taking the difference between (7) and (9) we finally get the estimation function

$$
\sum_{i=1}^{n} \frac{\dot{\mu}\left(T_{i-1} ; \theta\right)}{\sigma^{2}\left(T_{i-1} ; \theta\right)}\left(T_{i}-E_{\theta}\left(T_{i} \mid T_{i-1}\right)\right)=0 .
$$

Meanwhile, by equation (3) we have

$$
T_{i}=\left(T_{i-1}-M_{i-1}\right) e^{-\theta}+M_{i}+\int_{(i-1) \Delta}^{i \Delta} e^{-\theta \Delta} \sigma d W
$$

from which $E\left[T_{i} \mid T_{i-1}\right]=\left(T_{i-1}-M_{i-1}\right) e^{-\theta \Delta}+M_{i}$. It follows the Itô integration property that $E\left(\int_{(i-1) \Delta}^{i \Delta} e^{-\theta \Delta} \sigma d W\right)=$ 0 . Now it is possible to get the unique zero solution of (10) by substituting $E\left[T_{i} \mid T_{i-1}\right]$ in (10) as

$$
\theta=-\log \left(\frac{\sum_{i=1}^{n} \frac{M_{i-1}-T_{i-1}}{\sigma_{i-1}^{2}}\left(T_{i}-M_{i}\right)}{\sum_{i=1}^{n} \frac{M_{i-1}-T_{i-1}}{\sigma_{i-1}^{2}}\left(T_{i-1}-M_{i-1}\right)}\right)
$$

where $\sigma$ is obtained through (5).

\section{EXPERIMENT}

In this section, we use data generated from another modeling of human core temperature developed in [10] and [11] to estimate our parameters.

Based on [10], [11] core temperature may be expressed as

$$
y(t)=\mu+s(t)+v(t)
$$

where $\mu$ is the mean temperature, $s(t)$ is the circadian component and the noise $v(t)$ represents thermoregulatory response and other non-circadian perturbations. Moreover, $s(t)$ is the solution of the van der Pol equation of second order with

$$
s(t)=a(t) \cos \left(\omega t+\psi_{0}\right)-\epsilon \frac{a(t)}{8 \gamma^{2}} \sin \left(3 \omega t+3 \psi_{0}\right)+O\left(\epsilon^{2}\right)
$$

, where $\omega=\frac{2 \pi}{24}$ since we assume period is 24 hours, $\epsilon$ is internal stiffness parameter, and $\psi_{0}$ is the phase angle, $a(t)$ represents the dynamic amplitude of the circadian pacemaker satisfying

$$
\frac{d a(t)}{d t}=\frac{1}{2} \epsilon \omega\left[a(t)-\frac{a(t)^{3}}{\gamma^{2}}\right] .
$$

The function $v(t)$ is approached with $\mathrm{AR}(1)$ process as follows

$$
v(t)=\rho v(t-1)+\eta(t)
$$

where $\rho$ is the coefficient of $\operatorname{AR}(1)$ process and $\eta(t)$ is an independent Gaussian random variable with 0 mean and variance $\sigma^{2}$.

Table I presents the parameters for three simulated experiments. These parameters were obtained in [10], [11] from real world data. The mean temperature $\mu$ is assumed to be $98.6^{\circ} \mathrm{F}$ or so. And the data is generated with intervals of 20 minutes.

Each of the three simulations leveraging the parameters given in Table I are presented in Fig. 1, Fig. 2, and Fig. 3.

We use these simulated data as our ground truth to obtain parameters for the stochastic differential equation (1) based on the parameter estimation method in Section III. (Table II) 
FIG. 1: Regenerated Experiment 1

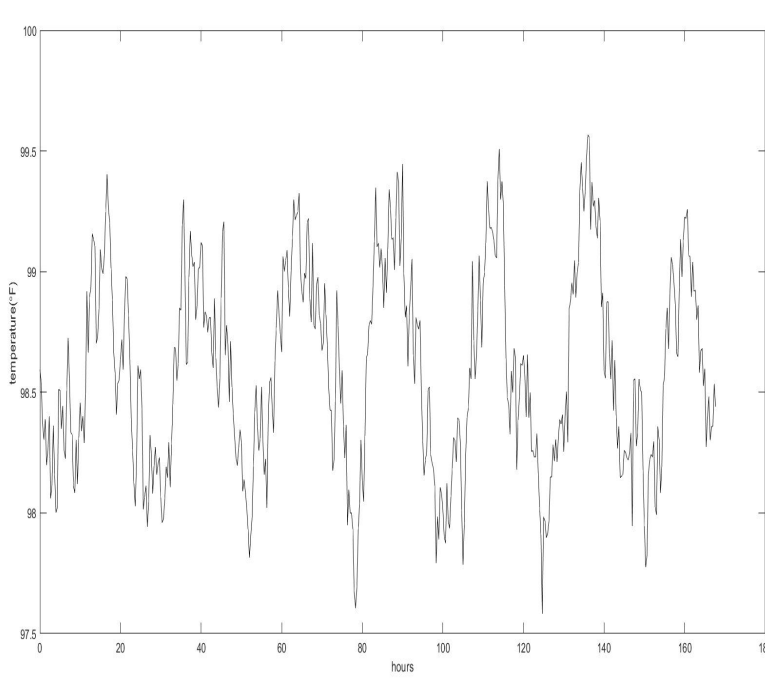

FIG. 2: Regenerated Experiment 2

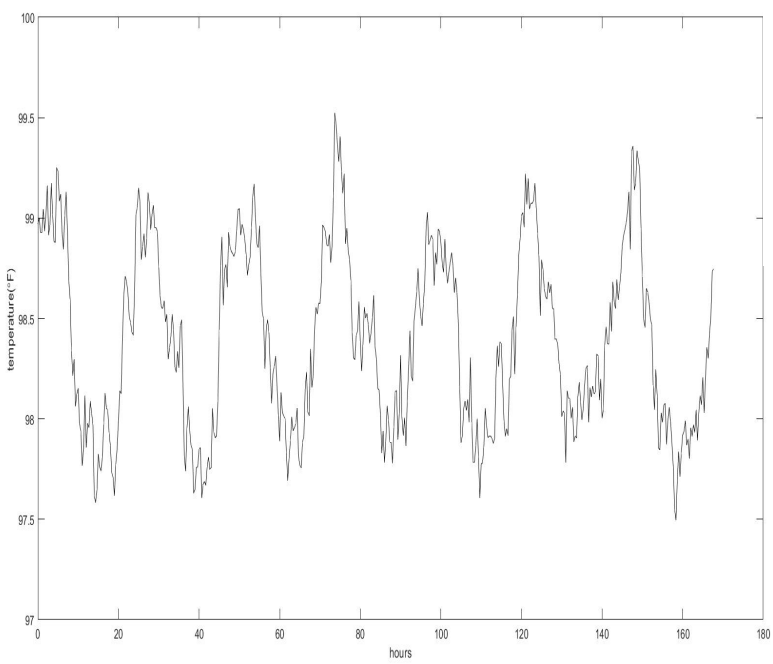

FIG. 3: Regenerated Experiment 3

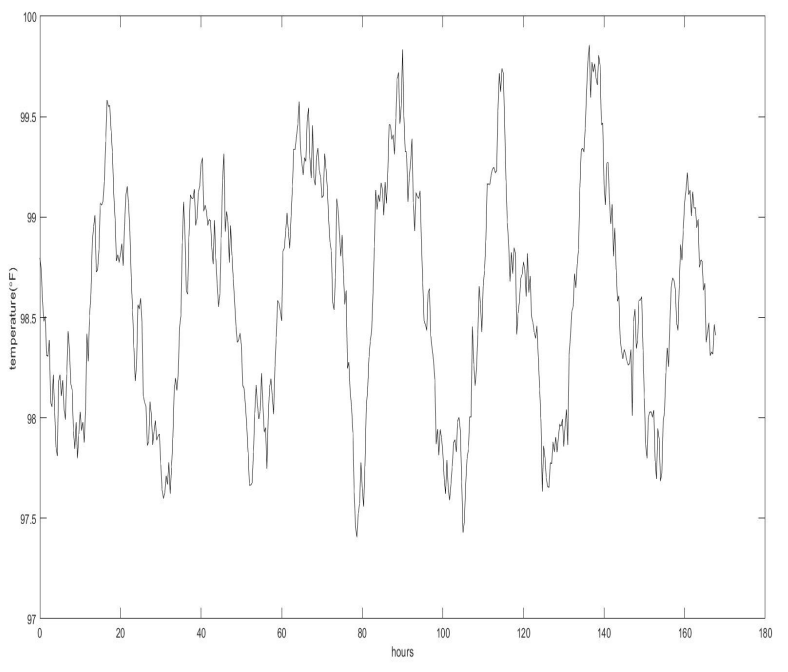

TABLE I: Experiments parameters corresponding to Fig.1, Fig.2, Fig. 3 respectively

\begin{tabular}{|c||c|c|c|c|c|c|c|}
\hline $\begin{array}{c}\text { Experiment } \\
\text { No. }\end{array}$ & $\begin{array}{c}\text { Mean } \\
\text { Temp. }\end{array}$ & $a_{0}$ & $\epsilon$ & $\gamma$ & $\psi_{0}$ & $\rho$ & $\sigma^{2}$ \\
\hline 1 & 98.6 & 0.40 & 0.023 & 0.500 & 2 & 0.7 & 0.026 \\
2 & 98.4 & 0.66 & 0.015 & 0.601 & -0.84 & 0.75 & 0.017 \\
3 & 98.0 & 0.70 & 0.200 & 0.438 & 1.50 & 0.90 & 0.020 \\
\hline
\end{tabular}

TABLE II: SDE parameters corresponding to SDE simulations in Fig.7, Fig.8, Fig.9 respectively

\begin{tabular}{|c|c|c|c|}
\hline $\begin{array}{c}\text { Experiment } \\
\text { No. }\end{array}$ & $\theta$ & $\sigma_{1}$ & $\sigma_{2}$ \\
\hline \multirow{7}{*}{1} & \multirow{7}{*}{0.4193} & 0.0307 & 0.0281 \\
\hline & & 0.0346 & 0.0243 \\
\hline & & 0.0247 & 0.0242 \\
\hline & & 0.0372 & 0.0285 \\
\hline & & 0.0331 & 0.0275 \\
\hline & & 0.0301 & 0.0241 \\
\hline & & 0.0303 & 0.0202 \\
\hline \multirow{7}{*}{2} & \multirow{7}{*}{0.3514} & 0.0201 & 0.0177 \\
\hline & & 0.0236 & 0.0154 \\
\hline & & 0.0164 & 0.0158 \\
\hline & & 0.0237 & 0.0187 \\
\hline & & 0.0219 & 0.0179 \\
\hline & & 0.0184 & 0.0157 \\
\hline & & 0.0208 & 0.0131 \\
\hline \multirow{7}{*}{3} & \multirow{7}{*}{0.1659} & 0.0234 & 0.0203 \\
\hline & & 0.0242 & 0.0178 \\
\hline & & 0.0178 & 0.0177 \\
\hline & & 0.0282 & 0.0211 \\
\hline & & 0.0244 & 0.0200 \\
\hline & & 0.0222 & 0.0179 \\
\hline & & 0.0211 & 0.0155 \\
\hline
\end{tabular}

In Fig. 4, Fig.5, Fig.6, $\sigma 1$ and $\sigma 2$ are compared graphically.

In Fig 7, Fig 8, Fig 9, SDEs of 3 Experiments are simulated (cf. [12]) at a numerical cost of $\triangle w=\Delta t * \varepsilon$, where we set $\triangle t=1 / 3$ hour and $\varepsilon$ is a standard normal distribution. Notably, the numerical cost does not have have a significant effect on the results of the simulation. In Fig. 10 and Fig. 11, the simulations with $\triangle t=1 / 60$ hour and $\triangle t=1 / 1000$ hour of Experiment 1 are displayed.

\section{CONCLUSION}

In this work, a mean-reverting SDE model, which is extensively used in industry, is introduced to simulate generic human body temperature. Then we leverage simulated data from an established model [10], [11] as our ground truth. The method of the present manuscript compares favorably with that of the established model.

Even though both models are dynamic with diffusion or perturbation the two models have their distinct advantages. Our reference model [10], [11] is the solution of van der Pol equation concerning the periodic trend, and it obtains the ability to express more detail (such as dynamic amplitude) in temperature variation. Also the established model from [10], [11] has the advantage inherent in its expression as an autoregressive model. Remarkably, our model may be easily modified to suit other body temperature areas (e.g. axilla, mouth, rectum, etc.), and has fewer parameters and a simpler expression.

However, there is a limitation to the SDE model presented in this manuscript. Even though our model follows the general 
trend of temperature fluctuation, it is lacking in terms of the violation $\sigma$. This violation has been discussed in other contexts where this method has been employed (cf. [5]). Future efforts will be directed towards the resolution of this limitation.

\section{ACKNOWLEDGMENT}

Hongliang Wang would like to express his appreciation with his wife, Shimin Zhang, who not only provides a tremendous amount of insight but also great inspiration. The authors appreciate the revision and advices from Dr. Joel Rosenfeld.

\section{REFERENCES}

[1] Mackowiak, Philip A. and Wasserman, Steven S. and Levine, Myron M., A Critical Appraisal of $98.6^{\circ} \mathrm{F}$, the Upper Limit of the Normal Body Temperature, and Other Legacies of Carl Reinhold August Wunderlich, JAMA, 268(12):1578-1580, 1992.

[2] Kelly Greg, Body temperature variability (Part 1): a review of the history of body temperature and its variability due to site selection, biological rhythms, fitness, and aging. Altern Med Rev, 11(4):278-293, 2006

[3] Bhowan A, Honours Project: Temperature Derivatives. 2003.

[4] Roberto Refinetti, The circadian rhythm of body temperature, Behavior Research Methods, 24(1):28-36, 1992.

[5] Peter Alaton, Boualem Djehiche, David Stillberger, On modelling and pricing weather derivatives, Applied Mathematical Finance, 9(1), 2002

[6] Brockwell, P.J. and Davis, R.A., Time Series: Theory and Methods, 2nd ed, Springer, 1990.

[7] Bo Martin Bibby, Michael Sorensen, Martingale Estimation Functions for Discretely Observed Diffusion Processes, Bernoulli, 1(1/2), 1995.

[8] Danielle, Florens-zmirou, Approximate discrete-time schemes for statistics of diffusion processes, Statistics, 20(4):547-557, 1989.

[9] Pederson, Asger Roer, A New Approach to Maximum Likelihood Estimation for Stochastic Differential Equations Based on Discrete Observations, Scandinavian Journal of Statistics, 22(1):55-71, 1995.

[10] Premananda Indic, Emery N Brown, Characterizing the amplitude dynamics of the human core-temperature circadian rhythm using a stochastic-dynamic model, Journal of Theoretical Biology, 239(4):499506, 2006.

[11] Emery N. Brown, Yong Choe, Harry Luithardt and Charles A. Czeisler, A statistical model of the human core-temperature circadian rhythm, Am J Physiol Endocrinol Metab, 279(3):E669-E683, 2000.

[12] Desmond J. Higham, An algorithmic Introduction to Numerical Simulation of stochastic Differential Equations, SIAM Review, 43(3):525-546, 2001
FIG. 4: $\sigma$ of Experiment 1

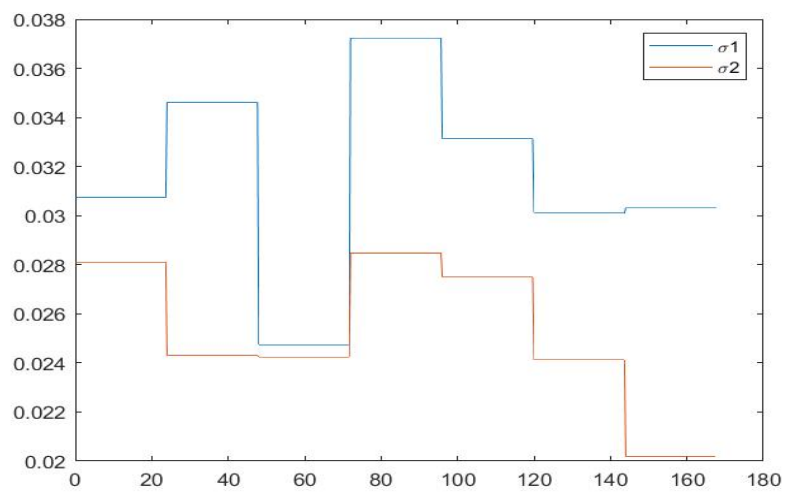

FIG. 5: $\sigma$ of Experiment 2

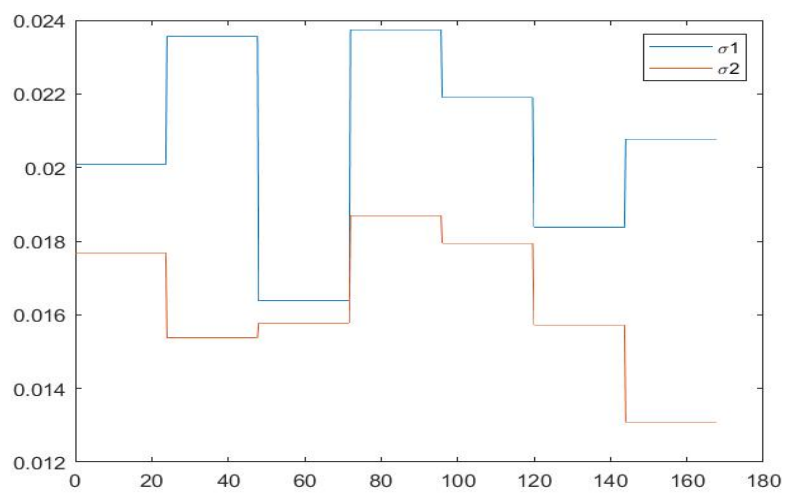

FIG. 6: $\sigma$ of Experiment 3

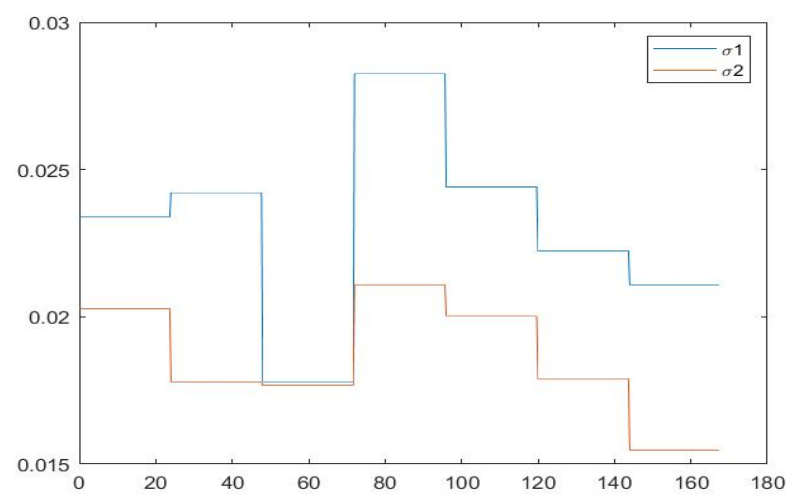


FIG. 7: SDE Simulate of Experiment 1

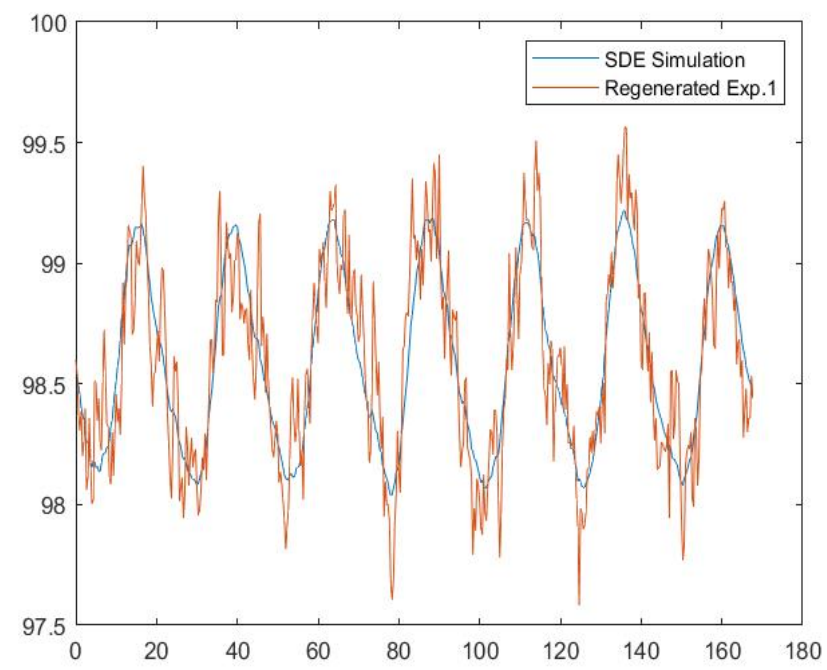

FIG. 8: SDE Simulate of Experiment 2

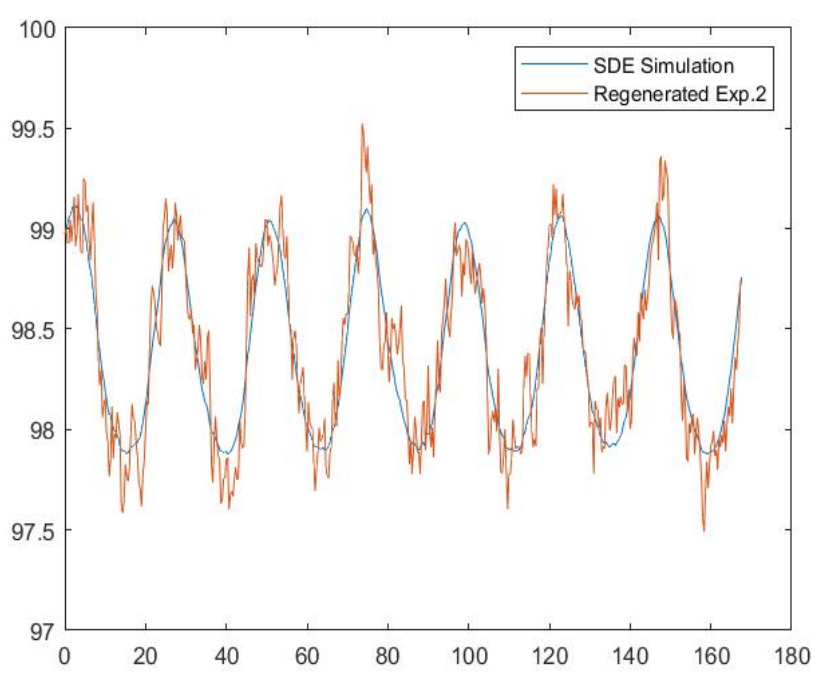

FIG. 9: SDE Simulate of Experiment 3

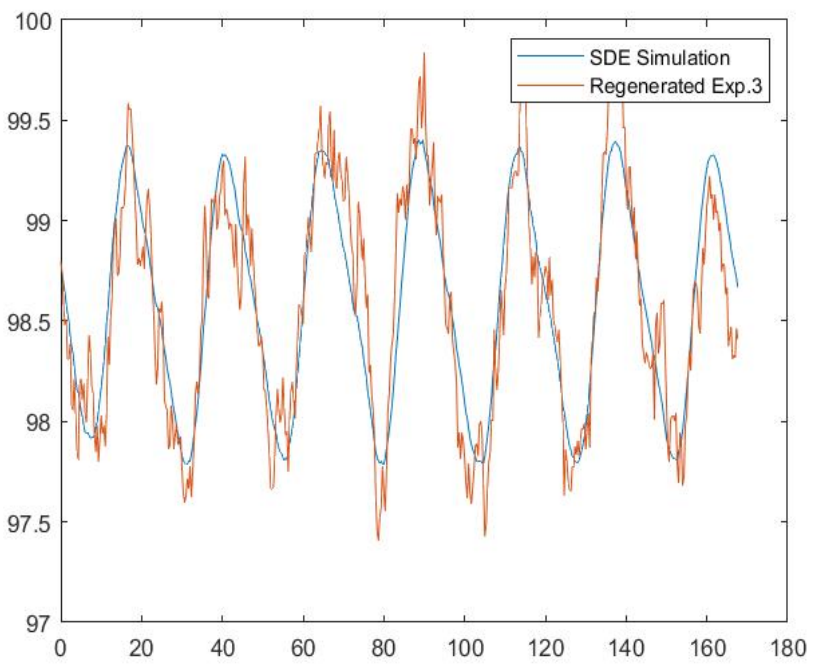

FIG. 10: SDE Simulate of Experiment 1 with $\triangle t=1 / 60$ hour

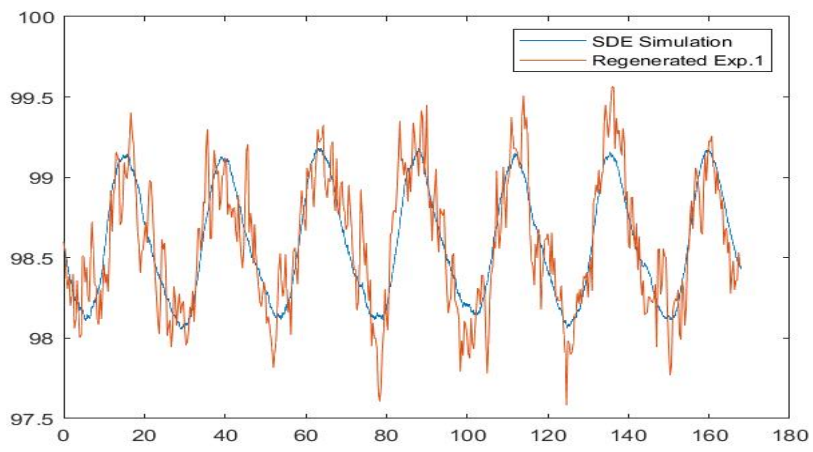

FIG. 11: SDE Simulate of Experiment 1 with $\triangle t=1 / 1000$ hour

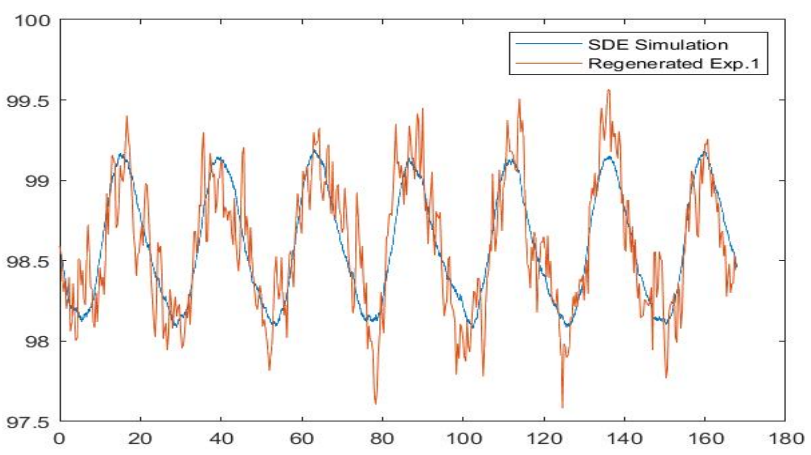

